[Article]

\title{
一个高粘弹的阴离子蠕虫胶束体系
}

\author{
谢丹华 ${ }^{1}$ 赵剑曦 ${ }^{1, *}$ 刘 琳 $^{1}$ 游 毅 ${ }^{1}$ 魏西莲 ${ }^{2}$ \\ ('福州大学化学化工学院, 胶体与界面化学研究所, 福州 350108; ${ }^{2}$ 聊城大学化学化工学院化学系, 山东聊城 252059)
}

摘要: 以频率扫描和稳态剪切实验研究了 $140 \mathrm{mmol} \cdot \mathrm{L}^{-1}$ 羧酸盐 $\mathrm{gemini}$ 表面活性剂 $\left(\mathrm{C}_{14} \mathrm{Q}_{2} \mathrm{C}_{14}\right)$ 在 $100 \mathrm{mmol}$. $\mathrm{L}^{-1} \mathrm{NaBr}$ 条件下溶液的流变行为. 在低剪切频率时, 溶液呈现出具有单一松弛时间特性的 Maxwell 流体行为. 通过活的高分子模型(living polymer model)分析, $\mathrm{C}_{14} \mathrm{~d}_{2} \mathrm{C}_{14}$ 体系在 $25^{\circ} \mathrm{C}$ 时形成了很长的蠕虫胶束(3.6-6.8 $\mu \mathrm{m}$ ). 冷冻透射电镜也观察到蠕虫胶束的形成. 这些胶束相互缠绕, 形成了很黏稠的溶液 (零剪切粘度高达 $\left.1.10 \times 10^{4} \mathrm{~Pa} \cdot \mathrm{s}\right)$, 外观呈现胶状. 随着温度升高至 $70^{\circ} \mathrm{C}$, 体系的相对粘度仍旧保持很高 $\left(1.8 \times 10^{4}\right)$, 这在阴离子 表面活性剂蠕虫胶束溶液中是很少见的. 体系的流动活化能 $\left(E_{\mathrm{a}}\right)$ 约为 $(141 \pm 5) \mathrm{kJ} \cdot \mathrm{mol}^{-1}$. 利用动态光散射测定了 $\mathrm{C}_{14} \phi_{2} \mathrm{C}_{14}$ 聚集体的尺寸分布, 证实了这个表面活性剂在 5-10 mmol. $\mathrm{L}^{-1}$ 的低浓度时生成了约 $100 \mathrm{~nm}$ 的大聚集 体, 这些大聚集体随着表面活性剂浓度的增加很容易转化成棒状直至蠕虫胶束.

关键词：羧酸盐 gemini 表面活性剂; 对联苯二酚联接链；蠕虫胶束； 高粘弹性； 温度效应; 胶束形成机理

中图分类号: $\mathrm{O} 648$

\section{A Highly Viscoelastic Anionic Wormlike Micellar System}

\author{
XIE Dan-Hua $^{1} \quad$ ZHAO Jian-Xi' ${ }^{1, *} \quad$ LIU Lin ${ }^{1} \quad$ YOU Yi ${ }^{1} \quad$ WEI Xi-Lian ${ }^{2}$ \\ ('Institute of Colloid and Interface Chemistry, College of Chemistry and Chemical Engineering, Fuzhou University, \\ Fuzhou 350108, P. R. China; ${ }^{2}$ Department of Chemistry, College of Chemistry and Chemical Engineering, \\ Liaocheng University, Liaocheng 252059, Shandong Province, P. R. China)
}

\begin{abstract}
The rheological behavior of carboxylate gemini surfactant $O, O^{\prime}$-bis(sodium 2tetradecylcarboxylate)- $p$-dibenzenediol (referred to as $\mathrm{C}_{14} \phi_{2} \mathrm{C}_{14}$ ) with a concentration of $140 \mathrm{mmol} \cdot \mathrm{L}^{-1}$ in aqueous solution in the presence of $100 \mathrm{mmol} \cdot \mathrm{L}^{-1} \mathrm{NaBr}$ was investigated by frequency sweep and steady rate sweep measurements. The solution showed the characteristics of a Maxwell fluid with a single stress relaxation time at low shear frequencies. Analysis by the living polymer model indicated that $\mathrm{C}_{14} \phi_{2} \mathrm{C}_{14}$ formed long $(3.6-6.8 \mu \mathrm{m})$ wormlike micelles at $25^{\circ} \mathrm{C}$, which were observed by cryo-transmission electron microscopy (cryo-TEM). The micelles were entangled with each other, resulting in a very viscous solution (the zero-shear viscosity was as high as $1.10 \times 10^{4} \mathrm{~Pa} \cdot \mathrm{s}$ ) that looked like a gel. On raising the temperature to $70^{\circ} \mathrm{C}$, the relative viscosity was still as high as $1.8 \times 10^{4}$, which is very rare for anionic wormlike micelle systems. The flow activation energy $\left(E_{\mathrm{a}}\right)$ was estimated to be $(141 \pm 5) \mathrm{kJ} \cdot \mathrm{mol}^{-1}$. The size distribution of $C_{14} \phi_{2} C_{14}$ was determined by dynamic light scattering. Large aggregates with a size of $\sim 100 \mathrm{~nm}$ were observed at low concentrations of $5-10 \mathrm{mmol} \cdot \mathrm{L}^{-1}$. These large aggregates readily transformed into rodlike and then wormlike micelles as the surfactant concentration increased.
\end{abstract}

Key Words: Carboxylate gemini surfactant; $\quad$-Dibenzenediol spacer; Wormlike micelle; High viscoelasticity; Temperature effect; Micelle formation mechanism

Received: February 4, 2013; Revised: April 24, 2013; Published on Web: April 25, 2013.

*Corresponding author. Email: jxzhao.colloid@fzu.edu.cn; Tel: +86-591-22866338.

The project was supported by the National Natural Science Foundation of China (20873024, 21273040).

国家自然科学基金(20873024, 21273040)资助项目

(C) Editorial office of Acta Physico-Chimica Sinica 


\section{Introduction}

Upon the addition of salts, cationic cetyltrimethylammonium halides $\left(\mathrm{C}_{16} \mathrm{TAX}\right)$ in aqueous solution can self-assembly into flexible threadlike (wormlike) micelles with the length even on the order of a few microns. ${ }^{1,2}$ At high concentrations these wormlike micelles entangle each other to form a transient network, which sharply increases the viscoelasticity of the solution. Comparatively, anionic wormlike micelles have advantage over cationic counterparts in many applications, including enhanced oil recovery. ${ }^{3}$ Moreover, anionic systems tend to be biodegradable and less toxic compared with cationic ones. But the formation of anionic wormlike micelles is not common since the addition of salts often interacts strongly with anionic surfactant and results in precipitate. Therefore, only a few anionic wormlike micelles were formed by conventional singlechain surfactants such as sodium alkyl sulfate ${ }^{4-9}$ or sodium dodecyltrioxylene sulfate ${ }^{10-14}$ in the presence of additives. What's more, these anionic micelles were not as long as the cationic ones and hence the anionic systems often followed a poor property (low viscoelasticity). For example, in the reported anionic wormlike micellar system consisted of SDS, the zero-shear viscosity of the solution only had a very low value of $\sim 1 \mathrm{~Pa} \cdot \mathrm{s}{ }^{5}$

Many efforts have been made to overcome this difficulty, in which the most valuable test was for the gemini surfactants. For example, Acharya et al..$^{15}$ tried to construct the wormlike micelles using a carboxylate gemini surfactant that has no spacer (designated as GS) mixed with the nonionic surfactants with a short poly(oxyethylene) chain $\left(\mathrm{C}_{m} \mathrm{E}_{n}\right.$, where $m, n=12,3 ; 12$, $4 ; 16,4$; respectively). They found that $\mathrm{C}_{12} \mathrm{E}_{3}$ very effectively promoted the formation of long wormlike micelles and as a result, the maximum viscosity of the solution attained about $10^{4}$ $\mathrm{Pa} \cdot \mathrm{s}$. Recently, we reported a novel anionic wormlike micelle system constructed by carboxylate gemini surfactants with an azobenzene spacer (designated as $\mathrm{C}_{m}(\mathrm{azo}) \mathrm{C}_{m}$, where $m$ represented the number of the carbon atoms in each alkyl tail and was 10,12 , and 14 , respectively, see Scheme $1(\mathrm{a})) .^{16,17}$ The azobenzene spacer has a stretched length of more than $1 \mathrm{~nm}$, which makes the head-group of $\mathrm{C}_{m}(\mathrm{azo}) \mathrm{C}_{m}$ including two carboxyl groups and a spacer quite large. This seems to say that $\mathrm{C}_{m}(\mathrm{azo}) \mathrm{C}_{m}$ is hard to form threadlike aggregates with a low surface curvature according to the molecular packing theory. ${ }^{18}$ Very interestingly, however, all these surfactants formed wormlike micelles. The mechanism has been attributed to the rigid

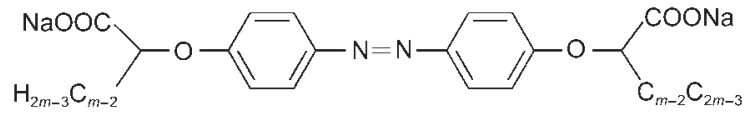

(a) $\mathrm{C}_{m}($ azo $) \mathrm{C}_{m}(m=10,12,14)$

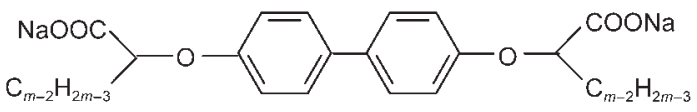

(b) $\mathrm{C}_{m} \phi_{2} \mathrm{C}_{m}(m=10,12,14)$

Scheme 1 Chemical structures of carboxylate gemini surfactants with long rigid spacer characteristic of the azobenzene spacer, which restrained the two alkyl tails within a gemini molecule drawing close and yielded the pseudo volume between them and thus increased the packing parameter, $P^{16,17}$ This finding strongly suggests that the gemini surfactants with a long rigid spacer may be good candidates for forming anionic wormlike micelles.

Very recently, we synthesized a new family of carboxylate gemini surfactants also with a long aromatic spacer, $O, O^{\prime}$ bis(sodium 2-alkylcarboxylate)-p-dibenzenediol, referred to as $\mathrm{C}_{m} \phi_{2} \mathrm{C}_{m}(m=10,12,14$, Scheme 1(b)). Surprisingly, we found that $\mathrm{C}_{12} \phi_{2} \mathrm{C}_{12}$ followed a new mechanism of aggregation different from general core-shell micelle formation. ${ }^{19}$ In dilute solution, $\mathrm{C}_{12} \phi_{2} \mathrm{C}_{12}$ formed large network-like aggregates. This behavior was attributed to an extending configuration of $\mathrm{C}_{12} \phi_{2} \mathrm{C}_{12}$ with the two alkyl tails stretching towards the solution due to the rigidity of the long spacer. Thus the large network-like aggregate formation was an inevitable outcome of spontaneously reducing the energy of the system. Due to the columnar-like molecular geometry of $\mathrm{C}_{12} \phi_{2} \mathrm{C}_{12}$, the network-like aggregates were very easily transformed into rod-like micelles with slightly increasing surfactant concentration and finally the wormlike micelles were formed. By this mechanism of aggregation, a new approach to constructing highly viscoelastic anionic wormlike micellar systems is perhaps revealed. In this paper, we report the wormlike micellar solution formed by $\mathrm{C}_{14} \phi_{2} \mathrm{C}_{14}$ that has the longest alkyl tails in this family so as to further understand this new constructing way.

\section{Experimental}

\subsection{Materials}

Sodium bromide (NaBr, purity $>99 \%$, Beijing Chemical Reagents Co.) was used as received. The water used was Milli-Q grade with a resistivity of $18.2 \mathrm{M} \Omega \cdot \mathrm{cm}$.

$O, O^{\prime}$-bis(sodium 2-tetradecylcarboxylate)- $p$-dibenzenediol (referred to as $\mathrm{C}_{14} \phi_{2} \mathrm{C}_{14}$ ) was synthesized in our laboratory according to the following routes (Scheme 2). All reagents used were purchased from Sinopharm Chemical Reagent Co., Ltd. (China).

Methyl 2-bromotetradecanoate was prepared according to our previous work. ${ }^{17}$

Synthesis of $\mathrm{MC}_{14} \phi_{2} \mathrm{C}_{14} \mathrm{M}$. The potassium carbonate $(9.7 \mathrm{~g}$, $70 \mathrm{~mol}$ ) was added to a three-necked flask with $20 \mathrm{~mL} \mathrm{~N}, N$-dimethylformamide (DMF). 4,4'-Biphenol (3.7 g, $20 \mathrm{mmol}$ ) was dissolved in $10 \mathrm{~mL}$ DMF, and then this solution was added to the mixture dropwise. Methyl 2-bromotetradecanoate (19.3 g, $60 \mathrm{mmol}$ ) was added into the mixture secondly. The mixture was reacted under stirring at $80{ }^{\circ} \mathrm{C}$ overnight. The mixture was poured into $70 \mathrm{~mL}$ ice-water and extracted with petroleum ether $(3 \times 60 \mathrm{~mL})$. The combined organic layers were washed with deionized water. The petroleum ether was removed under reduced pressure. The crude product was recrystallized from petroleum ether three times to give $\mathrm{MC}_{14} \phi_{2} \mathrm{C}_{14} \mathrm{M}$ as white powder. 
(l)

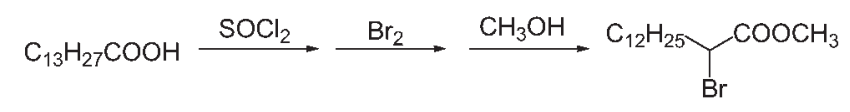

(II)
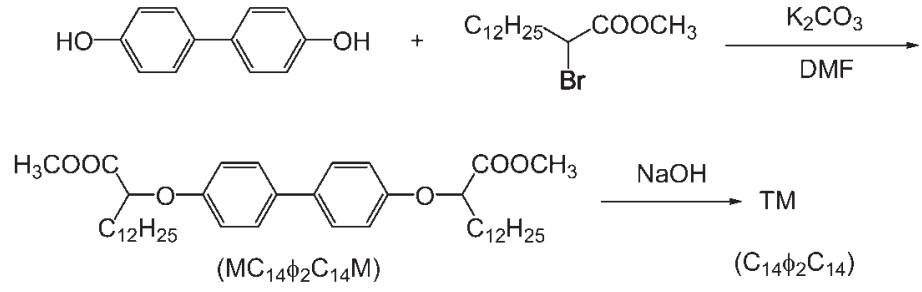

Scheme 2 Synthetic route of $\mathrm{C}_{14} \phi_{2} \mathrm{C}_{14}$

Synthesis of $\mathrm{C}_{14} \phi_{2} \mathrm{C}_{14}$. A mixture of $\mathrm{MC}_{14} \phi_{2} \mathrm{C}_{14} \mathrm{M}$ (6.4 g, 9.6 $\mathrm{mmol})$ and sodium hydroxide $(0.92 \mathrm{~g}, 23 \mathrm{mmol})$ in $500 \mathrm{~mL}$ of $95 \%$ ethanol was refluxed overnight. The resulting mixture was cooled to room temperature and centrifuged to afford the white precipitate. The precipitate was washed with ethanol several times and dried in vacuum to give the target compound as white powder. The final yield was $36.6 \%$ according to the quality of 4,4'-biphenol.

${ }^{1} \mathrm{H}$ NMR $\left(400 \mathrm{~Hz}, \mathrm{D}_{2} \mathrm{O}\right)$ for $\mathrm{C}_{14} \phi_{2} \mathrm{C}_{14}: \delta, 7.56(\mathrm{~d}, J=7.6 \mathrm{~Hz}$, 4H, H-Ar), 6.92 (d, J=7.6 Hz, 4H, H-Ar), 4.47 (br, 2H, CH), $1.90\left(\mathrm{~m}, 4 \mathrm{H}, \mathrm{CH}_{2}\right), 1.43-1.19\left(\mathrm{~m}, 4 \mathrm{H}, \mathrm{CH}_{2}\right), 1.05-0.82$ (m, $36 \mathrm{H}, \mathrm{CH}_{2}$ ), 0.43 (t, $J=6.4 \mathrm{~Hz}, 6 \mathrm{H}, \mathrm{CH}_{3}$ ).

Anal. Calcd. for $\left(\mathrm{C}_{40} \mathrm{H}_{60} \mathrm{Na}_{2} \mathrm{O}_{6}\right)(\%)$ : $\mathrm{C}, 70.35 ; \mathrm{H}, 8.86$; Found: C, 69.99; H, 8.65.

\subsection{Rheological measurements}

Rheological measurements were performed on a stress controlled rheometer (AR2000ex, TA instruments, USA) with conical concentric cylinders. The cone was made of standard ETC steel with the diameter of $40 \mathrm{~mm}$ and cone angle of $2^{\circ}$. The gap between the center of the cone and plate was $50 \mu \mathrm{m}$. At a designed temperature, the sample was kept for $5 \mathrm{~min}$ on the plate to reach the equilibrium before testing. A strain sweep was performed at a frequency of $6.28 \mathrm{rad} \cdot \mathrm{s}^{-1}(1 \mathrm{~Hz})$ before the test. A strain value was then decided to make sure of the sample in the linear viscoelastic region during the following oscillatory measurements.

\subsection{Cryogenic transmission electron microscopy (cryo-TEM)}

Cryo-TEM samples were prepared in a controlled environment vitrification system (CEVS) at $28^{\circ} \mathrm{C}$. A micropipet was used to load $5 \mu \mathrm{L}$ surfactant solution onto a lacey support TEM grid, which was held by tweezers. The excess solution was blotted with a piece of filter paper and the thin film was suspended on the mesh hole. After waiting for about $10 \mathrm{~s}$ to relax any stresses induced during the blotting, the samples were quickly plunged into a reservoir of liquid ethane (cooled by the nitrogen) at its melting temperature. The vitrified samples were then stored in the liquid nitrogen until they were transferred to a cryogenic sample holder (Gatan 626) and examined with a JEM 2200FS TEM $(200 \mathrm{keV})$ at about $-174{ }^{\circ} \mathrm{C}$. The phase contrast was enhanced by underfocus. The images were recorded on a Gatan multiscan CCD and processed with Digital Mi- crograph.

\subsection{Dynamic light scattering}

Dynamic light scattering (DLS) of micellar solutions was measured with a Brookhaven Instrument which was composed of a BI-200SM goniometer, a BI-9000AT digital correlator (522 channels) and a photomultiplier detector. The He-Ne laser with $15 \mathrm{~mW}$ power and $632.8 \mathrm{~nm}$ wavelength was used as the light source. The measurement temperature was controlled by a thermostatic circulator (Poly-sceience, USA) with an accuracy of $\pm 0.01{ }^{\circ} \mathrm{C}$. All solutions were filtered through $0.22 \mu \mathrm{m}$ Millipore filters into cylindrical light-scattering cells (od $=25$ $\mathrm{mm})$.

The intensity-intensity time correlation function $G^{(2)}(t, q)$ in the self-beating mode was measured, where $t$ is decay time and $q$ is scattering vector and equals to $4 \pi n / \lambda \sin (\theta / 2)$. The $G^{(2)}(t, q)$ was transformed into the electric field-electric field time correlation function $g^{(1)}(t, q)$ by Siegert formula:

$$
G^{(2)}(t, q)=A\left(1+\beta\left|g^{(1)}(t, q)\right|^{2}\right)
$$

where $A$ is the baseline, $\beta$ is a parameter depending on the coherence of the detection. The $g^{(1)}(t, q)$ was further related to the characteristic line-width $(\Gamma)$ distribution $G(\Gamma)$ by

$$
g^{(1)}(t, q)=\int_{0}^{\infty} G(\Gamma) \exp (-\Gamma t) \mathrm{d} \Gamma
$$

The $G(\Gamma)$ can be obtained by a Laplace inversion of $g^{(1)}(t, q)$ using the CONTIN program. The average line-width $\langle\Gamma\rangle$ was calculated according to

$$
\langle\Gamma\rangle=\int \Gamma G(\Gamma) \mathrm{d} \Gamma
$$

Furthermore, the apparent translational diffusive coefficient $D_{\text {app }}$ can be related to $<\Gamma>$ as $D_{\text {app }}=<\Gamma>/ q^{2}$. Thus, the apparent hydrodynamic radius of aggregate $\left(R_{\mathrm{h}, \mathrm{ppp}}\right)$ was obtained from the Stokes-Einstein equation

$$
R_{\mathrm{h}, \mathrm{ppp}}=\frac{k_{\mathrm{B}} T}{6 \pi \eta_{0} D_{\text {app }}}
$$

where $k_{\mathrm{B}}$ is the Boltzmann constant, $T$ is absolute temperature, and $\eta_{0}$ is the solvent viscosity.

\section{Results and discussion}

\subsection{Characteristic of wormlike micelles at $25^{\circ} \mathrm{C}$}

The aqueous system of $\mathrm{C}_{14} \phi_{2} \mathrm{C}_{14}\left(140 \mathrm{mmol} \cdot \mathrm{L}^{-1}\right) / \mathrm{NaBr}(100$ $\mathrm{mmol} \cdot \mathrm{L}^{-1}$ ) at $25^{\circ} \mathrm{C}$ has a gel-like appearance (Fig.1a). This is due to the formation of wormlike micelles, which entangle 

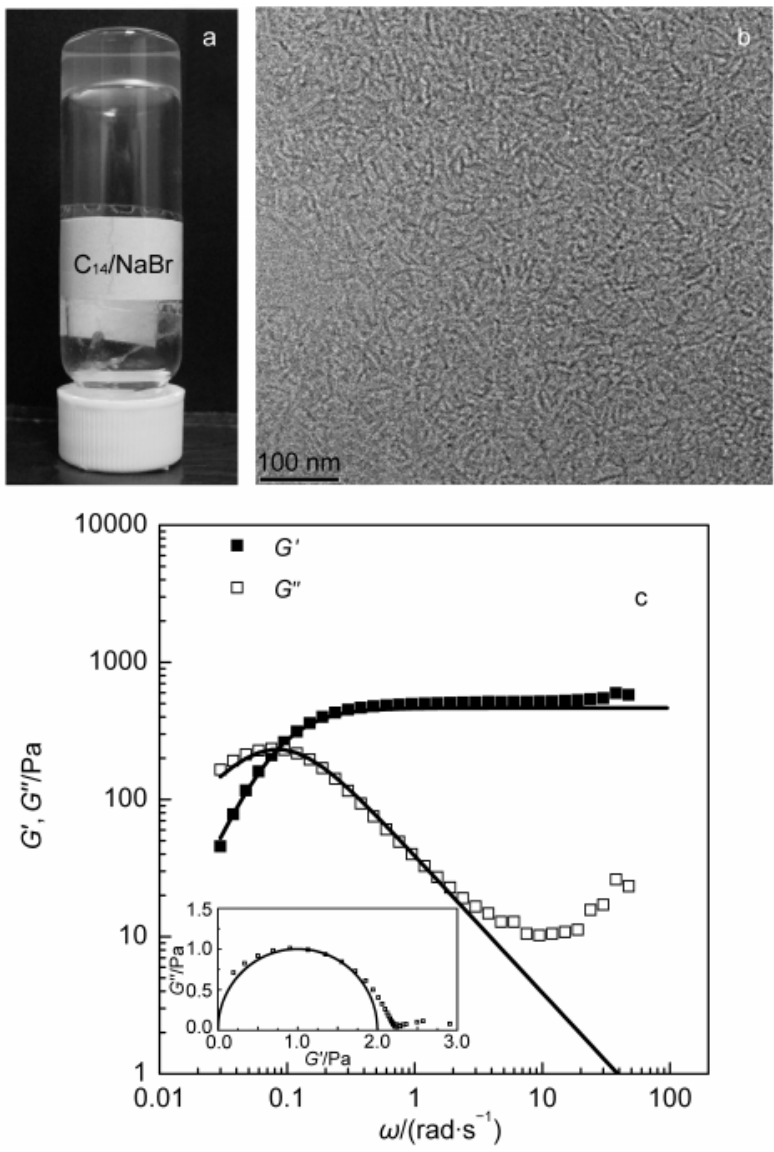

Fig.1 (a) Appearance of $\mathrm{C}_{14} \phi_{2} \mathrm{C}_{14}\left(140 \mathrm{mmol} \cdot \mathrm{L}^{-1}\right) / \mathrm{NaBr}$ $\left(100 \mathrm{mmol} \cdot \mathrm{L}^{-1}\right)$ aqueous system at $25^{\circ} \mathrm{C}$, (b) the cryo-TEM image, and (c) its variations of $G^{\prime}$ (filled symbols), $G^{\prime \prime}$ (open symbols) with sweep frequency $\omega$

The insert in figure $\mathrm{c}$ shows the Cole-Cole plot.

each other into a transient network as seen in the cryo-TEM image (Fig.1b). The result of frequency sweep measurement (Fig.1c) shows high elasticity, where elastic modulus $G^{\prime}$ exceeds viscous modulus $G^{\prime \prime}$ at sweep frequency $\omega>0.1 \mathrm{rad} \cdot \mathrm{s}^{-1}$. This system very well follows the Maxwell fluid behavior with a single stress relaxation time $\left(\tau_{\mathrm{R}}\right)^{20}$ as fitted (solid lines in Fig.1c) by the equations:

$$
\begin{aligned}
G^{\prime} & =\frac{\left(\omega \tau_{\mathrm{R}}\right)^{2}}{1+\left(\omega \tau_{\mathrm{R}}\right)^{2}} G_{\infty}^{\prime} \\
G^{\prime \prime} & =\frac{\omega \tau_{\mathrm{R}}}{1+\left(\omega \tau_{\mathrm{R}}\right)^{2}} G_{\infty}^{\prime}
\end{aligned}
$$

The corresponding Cole-Cole plot, i.e., elastic modulus $G^{\prime}$ against viscous modulus $G^{\prime \prime}$ (Fig.1c, insert), shows a perfect semicircular shape at low and medium frequencies, which is another indication for the Maxwell fluid behavior.

The $\tau_{\mathrm{R}}$ is estimated as $\omega_{\mathrm{c}}{ }^{-1}$, where $\omega_{\mathrm{c}}$ is the frequency at which two moduli are equal. ${ }^{21}$ On the increase in $\omega$, the $G^{\prime}$ attains a limiting value called a plateau modulus, $G_{\infty}^{\prime}$. The living polymer model proposed by Granek and $\mathrm{Cates}^{20}$ revealed two time scales of stress relaxation, namely, reptation time $\left(\tau_{\text {rер }}\right)$, which corresponds to the curvilinear diffusion of a chain of the mean length along its own contour, and breaking time $\left(\tau_{\mathrm{b}}\right)$.
When breaking occurs over the time scale of reptation $\left(\tau_{\mathrm{b}}<<\right.$ $\left.\tau_{\text {rep}}\right)$, as in a typical wormlike micellar system, the chain undergoes many breakages and recombinations before a chain segment relaxes by reptation. Thus the stress relaxation is characterized by a new time scale given by $\tau_{\mathrm{R}}=\left(\tau_{\mathrm{b}} \tau_{\text {rep }}\right)^{1 / 2}$, and the solution behaves as a Maxwell fluid with single relaxation time $\tau_{\mathrm{R}}$. The average scission time for the micelle, $\tau_{\mathrm{b}}$, is approximately equal to the inverse of $\omega$ corresponding to the minimum of $G_{\min }^{\prime \prime}$ in the high-frequency region. For the present system, the estimated $\tau_{\mathrm{b}}$ is $\sim 0.105 \mathrm{~s}$. This value allows us to estimate $\tau_{\text {rep }} \sim$ $1.35 \times 10^{3} \mathrm{~s}$ using $\tau_{\mathrm{R}}(11.9 \mathrm{~s})$, satisfying the expectation of $\tau_{\mathrm{b}}<<$ $\tau_{\text {rep. }}{ }^{20}$

The micellar contour length $(L)$ can be estimated by the relation $^{20}$

$$
\frac{G_{\min }^{\prime \prime}}{G_{\infty}^{\prime}} \approx \frac{l_{\mathrm{e}}}{L}
$$

where $l_{\mathrm{e}}$ is the average length between two entanglement points and $G_{\min }^{\prime \prime}$ is the minimum at $G^{\prime \prime}$ at high-frequency region. For a given $l_{\mathrm{e}}, L$ is inversely proportional to the ratio $G_{\min }^{\prime \prime} / G_{\infty}^{\prime}$. Thus, a smaller $G_{\min }^{\prime \prime} / G_{\infty}^{\prime}$ results in a longer $L$. So far, the smallest $G_{\min }^{\prime \prime} / G^{\prime}$ $\infty$ reported was 0.014 yielded in the mixed system of carboxylate gemini surfactant with no spacer (GS) and $\mathrm{C}_{12} \mathrm{E}_{3} \cdot{ }^{15}$ In this case, Acharya et al. ${ }^{15}$ have stressed that the added $\mathrm{C}_{12} \mathrm{E}_{3}$ played a very important role in promoting micellar growth. Another small $G_{\text {min }}^{\prime \prime} / G_{\infty}^{\prime}$ was 0.016 obtained from the system of traditional cationic surfactant having an unsaturated tail as long as 22 carbon atoms. ${ }^{22}$ For the present system, the $G_{\text {min }}^{\prime \prime} / G_{\infty}^{\prime}$ is about 0.022 . Even though this value is not as low as those mentioned above, it is produced in an anionic wormlike micellar system only upon the addition of simple salt and hence is very rare.

As a comparison, a typical value of $l_{\mathrm{e}}(80-150 \mathrm{~nm})$ for wormlike micelles can be adopted ${ }^{15}$ and thus $L$ corresponds to roughly $3.6-6.8 \mu \mathrm{m}$ for the present system at $25^{\circ} \mathrm{C}$. This length is indeed greatly longer than that of cetyltrimethylammonium bromide $\left(\mathrm{C}_{16} \mathrm{TABr}\right)$ micelle in the presence of $1.5 \mathrm{~mol}$. $\mathrm{L}^{-1} \mathrm{NaBr}^{23}$

\subsection{Zero-shear viscosity at $25^{\circ} \mathrm{C}$}

Fig. 2 shows the viscosity versus steady shear rate curve for the aqueous system of $\mathrm{C}_{14} \phi_{2} \mathrm{C}_{14}\left(140 \mathrm{mmol} \cdot \mathrm{L}^{-1}\right) / \mathrm{NaBr}(100$ $\left.\mathrm{mmol} \cdot \mathrm{L}^{-1}\right)$. The viscosity keeps unchanged at low shear rate re-

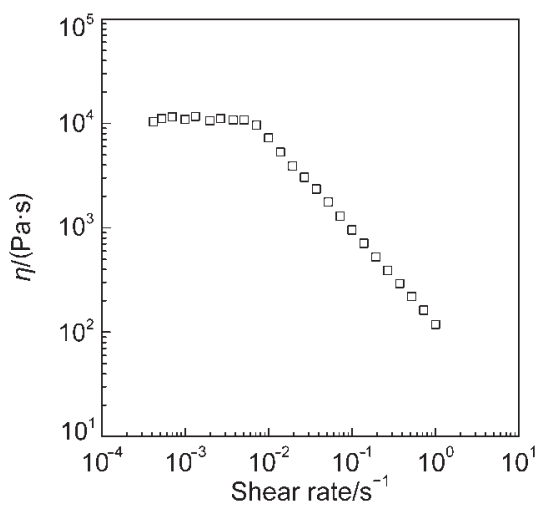

Fig.2 Variations of viscosity $(\eta)$ with the shear rate at $25^{\circ} \mathrm{C}$ 
gion and then decreases with increasing the shear rate (the shear thinning behavior) after a critical shear rate, $r_{\mathrm{c}}$. The shear thinning behavior can be taken as an evidence for the formation of wormlike micelles, ${ }^{24}$ which well corresponds to the indication of dynamic viscoelastic measurements. According to the Carreau model, the zero-shear viscosity $\eta_{0}$ is obtained to be $1.10 \times 10^{4} \mathrm{~Pa} \cdot \mathrm{s}$, meaning the relative viscosity of the wormlike micellar solution ( $\eta_{\mathrm{r}}$, given by $\eta_{\mathrm{o}} / \eta_{\mathrm{s}}$, where $\eta_{\mathrm{s}}$ is the viscosity of the solvent, water) to be as high as $10^{7}$. This is a very large value comparable with that obtained from the mixed system of gemini surfactant (GS) and $\mathrm{C}_{12} \mathrm{E}_{3} .^{15}$

\subsection{Mechanism of wormlike micelle formation}

As seen in Sections 3.1 and 3.2, $\mathrm{C}_{14} \phi_{2} \mathrm{C}_{14}$ can form wormlike micelles in aqueous solution, which is similar to the behavior of $\mathrm{C}_{14}(\mathrm{azo}) \mathrm{C}_{14}$ as reported previously. ${ }^{17}$

Fig.3 (left row) shows the intensity-fraction distribution of $\mathrm{C}_{14} \phi_{2} \mathrm{C}_{14}$ measured by DLS, in which the aggregates at 5 and 10 $\mathrm{mmol} \cdot \mathrm{L}^{-1}$ show quite large size $(\sim 100 \mathrm{~nm}$ in apparent hydrodynamic radius, $R_{\mathrm{h}, \text { app }}$ ) and narrow distribution. With increasing the surfactant concentration $(C)$, the mean $R_{\mathrm{h}}$ increases obviously at $C \geq 20 \mathrm{mmol} \cdot \mathrm{L}^{-1}$, indicating the rapid growth of micelles. This behavior is very analogous to that of $\mathrm{C}_{12} \phi_{2} \mathrm{C}_{12}$ which is another member of this family. Thus, as revealed in our previous work, ${ }^{19} \mathrm{C}_{14} \phi_{2} \mathrm{C}_{14}$ must form large network-like aggregates after

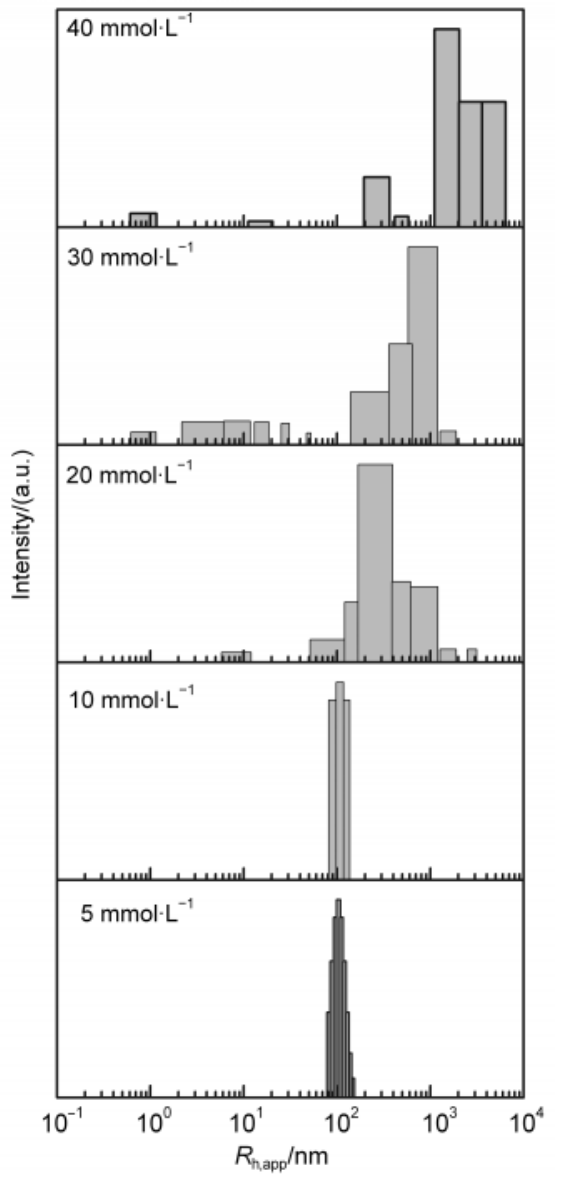

the critical micelle concentration $(\mathrm{cmc})$, and these network-like aggregates can be very easily transformed into rod-like micelles with slightly increasing the surfactant concentration and finally the wormlike micelles are formed.

However, it is very surprising that $\mathrm{C}_{14}($ azo $) \mathrm{C}_{14}$ shows the aggregation behavior different from $\mathrm{C}_{14} \phi_{2} \mathrm{C}_{14}$, although this surfactant also has a long aromatic spacer in its molecular structure. Fig.3 (right row) shows the scattering intensity of $\mathrm{C}_{14}(\mathrm{azo}) \mathrm{C}_{14}$ only distributes at small $R_{\mathrm{h}}$ values $(0.1-15 \mathrm{~nm})$ but a very wide range (polydispersity in size). The two cases $\left(\mathrm{C}_{14} \phi_{2} \mathrm{C}_{14}\right.$ and $\left.\mathrm{C}_{14}(\mathrm{azo}) \mathrm{C}_{14}\right)$ reflect the complicacy of gemini surfactants in self-assembly and sometimes these surfactants show completely different behaviors although their molecular structures are similar. A good understanding for their mechanisms needs further and deep investigations.

\subsection{Effect of temperature}

Fig.4a shows the viscoelastic spectra of this system at different temperatures. On the raise in temperature, the rheological behavior still shows semicircular shape in the Cole-Cole plots (Fig.4b) over the range of low and medium frequencies. Similarly, the characteristic parameters described above can be obtained according to living polymer model. ${ }^{20}$ For those cases where the $G^{\prime}$ does not give a constant limiting value, the $G_{\infty}^{\prime}$ may be estimated from the modulus value at $\omega_{\mathrm{c}}$, using the rela-

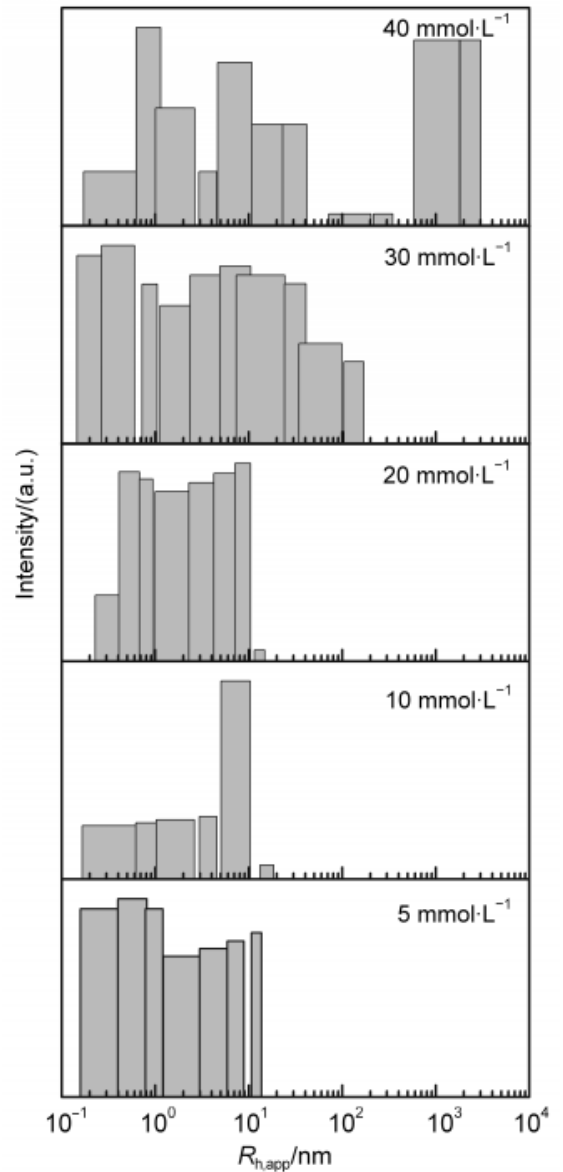

Fig.3 Intensity-fraction distributions measured at a detector angle $\theta=90^{\circ}$ and analyzed by CONTIN model for $\mathrm{C}_{14} \phi_{2} \mathrm{C}_{14}$ (left row) and $\mathrm{C}_{14}(\mathrm{azo}) \mathrm{C}_{14}$ (right row) at different concentrations 

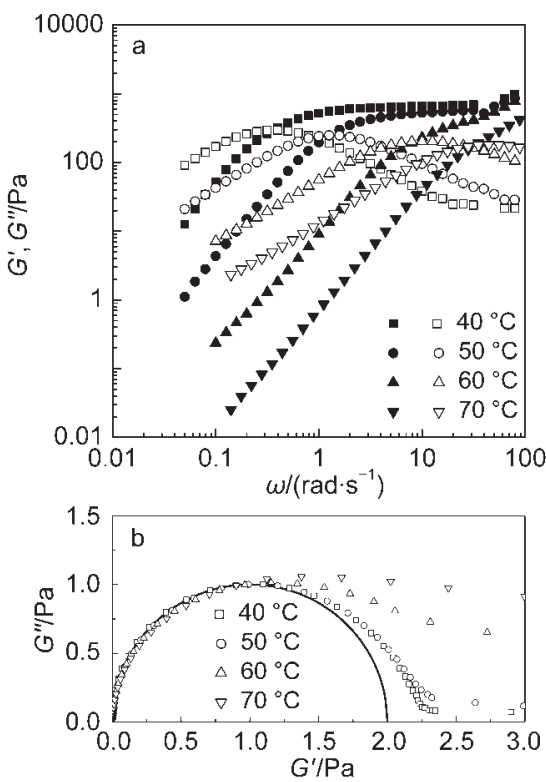

Fig.4 Viscoelastic spectra (a) and the normalized Cole-Cole plots (b) at different temperatures

tion $G_{\infty}^{\prime}=2 G_{\max }^{\prime \prime}$, where $G_{\max }^{\prime \prime}$ is the viscosity modulus at shear frequency $\omega_{\mathrm{c} .}{ }^{21}$ As seen from Table 1 , the $\tau_{\mathrm{R}}$ monotonically decreases over the entire temperature range, but $G_{\infty}^{\prime}$ shows an initial enhancement at low temperatures followed by a subsequent decrease at higher temperatures. Since $\tau_{\mathrm{R}}$ associates with the micellar length, ${ }^{15}$ the decrease in $\tau_{\mathrm{R}}$ indicates the wormlike micelles become shorter with increasing temperature. This is consistent with the estimation for the length from the ratio of $G_{\min }^{\prime \prime} / G_{\infty}^{\prime}$, which gives out the values of gradual decrease in $L$ (Table 1). For the wormlike micellar system undergoing stress relaxation by reptation, $G_{\infty}^{\prime}$ generally depends on the number density of the aggregates. ${ }^{15}$ The initial increase in $G_{\infty}^{\prime}$ over the temperature range of $25-40{ }^{\circ} \mathrm{C}$ suggests that the enhanced thermal motion promotes the contact between the micelles and thus yields more entanglements, which makes the system more viscoelastic. The following decrease in $G_{\infty}^{\prime}$ above $40{ }^{\circ} \mathrm{C}$ must be due to the shorter micelles as discussed above.

The zero-shear viscosity drown from Fig.5 has a value of $17.6 \mathrm{~Pa} \cdot \mathrm{s}$ at $70^{\circ} \mathrm{C}$, namely, its relative viscosity is $1.8 \times 10^{4}$. In general, with increasing temperature to $60{ }^{\circ} \mathrm{C}$, the $\eta_{\mathrm{r}}$ of wormlike micellar solution falls to a value below $10^{4}$. There are few, if any, examples of micellar solutions showing high viscosities $\left(\eta_{\mathrm{r}}>10^{4}\right)$ above $60^{\circ} \mathrm{C}$. The typical cases are the wormlike micel-

Table 1 Characteristic parameters of the wormlike micellar systems of $\mathrm{C}_{14} \phi_{2} \mathrm{C}_{14}\left(140 \mathrm{mmol} \cdot \mathrm{L}^{-1}\right) / \mathrm{NaBr}\left(100 \mathrm{mmol} \cdot \mathrm{L}^{-1}\right)$ at different temperatures

\begin{tabular}{ccccccc}
\hline$T /{ }^{\circ} \mathrm{C}$ & $\eta_{\mathrm{o}} /(\mathrm{Pa} \cdot \mathrm{s})$ & $\tau_{\mathrm{R}} / \mathrm{s}$ & $G_{\alpha}^{\prime} / \mathrm{Pa}$ & $\tau_{\mathrm{b}} / \mathrm{s}$ & $\tau_{\mathrm{rep}} / \mathrm{s}$ & $L / \mu \mathrm{m}$ \\
\hline 25 & $1.10 \times 10^{4}$ & 11.9 & 464.2 & 0.1050 & $1.35 \times 10^{3}$ & $3.6-6.8$ \\
40 & $1.73 \times 10^{3}$ & 2.81 & 584.8 & 0.0159 & $4.96 \times 10^{3}$ & $2.2-4.1$ \\
50 & 405 & 0.789 & 498.0 & 0.0138 & 44.9 & $1.4-2.6$ \\
60 & 85.7 & 0.120 & 401.4 & & & \\
70 & 17.6 & 0.032 & 351.6 & & & \\
\hline
\end{tabular}

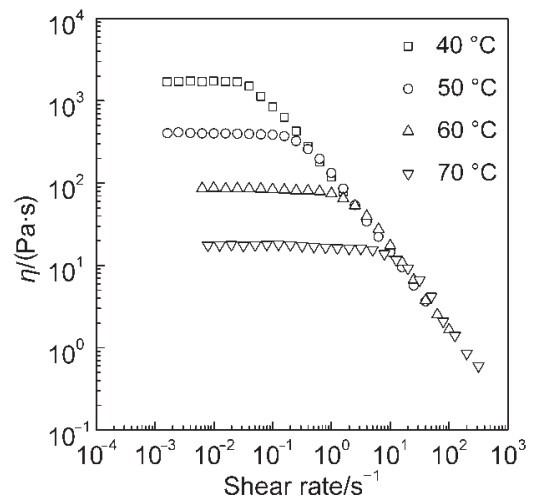

Fig.5 Variations of viscosity ( $\eta$ ) with the shear rate at different temperatures

lar solutions formed by cationic surfactants, erucyl bis(hydroxyethyl)methylammonium chloride (EHAC) and erucyl trimethylammonium chloride (ETAC), as reported by Raghavan and Kaler. ${ }^{22}$ The both surfactants bear a quite long $\left(\mathrm{C}_{22}\right)$ tail. Relying on the help of sodium salicylate or sodium chloride, the both systems retain high viscosity $\left(\eta_{\mathrm{r}}>10^{4}\right)$ up to ca $90{ }^{\circ} \mathrm{C}$. Compared with EHAC or ETAC, $\mathrm{C}_{14} \phi_{2} \mathrm{C}_{14}$ only has short tails (12 carbon atoms in each tail, Scheme 1(b)). Even so, the relative viscosity $\eta_{\mathrm{r}}$ still attains a rather large value at $70{ }^{\circ} \mathrm{C}$. This is very rare for the anionic wormlike micelle systems.

\subsection{Flow activation energy}

The variation of $\eta_{0}$ and $\tau_{\mathrm{R}}$ with temperature can be empirically described by Arrhenius relationships, indicating exponential reductions in these quantities: $:^{25,26}$

$$
\begin{aligned}
& \eta_{0}=G_{\infty}^{\prime} A \exp \left(E_{\mathrm{a}} / R T\right) \\
& \tau_{\mathrm{R}}=A \exp \left(E_{\mathrm{a}} / R T\right)
\end{aligned}
$$

where $E_{\mathrm{a}}$ is the flow activation energy, $R$ is the gas constant, and $A$ is a pre-exponential factor. Semilogarithmic plots of $\eta_{0}$ and $\tau_{\mathrm{R}} v s 1 / T$ (Fig.6) fall on straight lines within experimental error, being consistent with Eqs.(8) and (9). The slope gives out the flow activation energy $E_{\mathrm{a}}$ to be $(141 \pm 5) \mathrm{kJ} \cdot \mathrm{mol}^{-1}$. This value is comparable to that reported for other wormlike micelles. $^{22,24}$

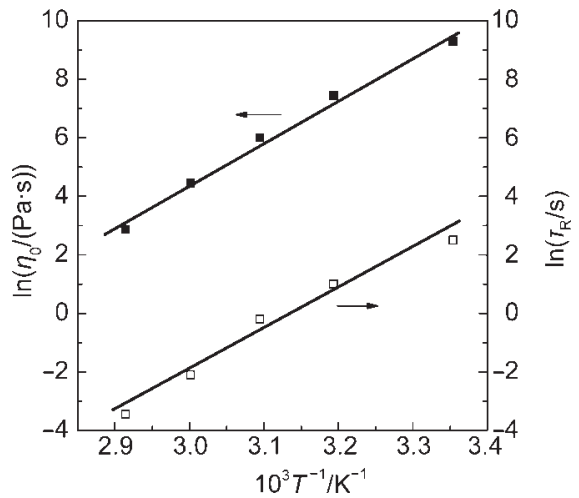

Fig.6 Arrhenius plots of the zero-shear viscosity $\left(\eta_{0}\right)$ and the relaxation time $\left(\tau_{\mathrm{R}}\right)$ vs $1 / T$ 


\section{Conclusions}

The present study exhibited an excellent anionic wormlike micelle system formed by carboxylate gemini surfactant $\mathrm{C}_{14} \phi_{2} \mathrm{C}_{14}$. This result again demonstrates that the gemini surfactants are very good candidates for the construction of wormlike micelles and also strongly suggests that based on the gemini surfactants, an effective approach may be developed to construct the anionic wormlike micelle systems with excellent viscoelastic properties.

\section{References}

(1) Dreiss, C. A. Soft Matter 2007, 3, 956. and references therein. doi: 10.1039/b705775j

(2) Yang, J. Curr. Opin. Colloid Interface Sci. 2002, 7, 276. and references therein. doi: 10.1016/S1359-0294(02)00071-7

(3) Maitland, G. C. Curr. Opin. Colloid Interface Sci. 2000, 5, 301. doi: 10.1016/S1359-0294(00)00069-8

(4) Magid, L. J.; Li, Z.; Butler, P. D. Langmuir 2000, 16, 10028. doi: 10.1021/la0006216

(5) Hassan, P. A.; Raghavan, S. R.; Kaler, E. W. Langmuir 2002, 18, 2543. doi: 10.1021/la011435i

(6) Arleth, L.; Bergstrom, M.; Pedersen, J. S. Langmuir 2002, 18, 5343. doi: 10.1021/la015693r

(7) Nakamura, K.; Shikata, T. Langmuir 2006, 22, 9853. doi: 10.1021/la061031w

(8) Acharya, D. P.; Sato, T.; Kaneko, M.; Singh, Y.; Kunieda, H. J. Phys. Chem. B 2006, 110, 754. doi: 10.1021/jp054631x

(9) Lu, T.; Xia, L. G.; Wang, X. D.; Wang, A. Q.; Zhang, T. Langmuir 2011, 27, 9815. doi: 10.1021/la2018709

(10) Mu, J. H.; Li, G. Z. Chem. Phys. Lett. 2001, 345, 100. doi: 10.1016/S0009-2614(01)00799-0

(11) Mu, J. H.; Li, G. Z. Colloid Polym. Sci. 2001, 279, 872. doi: $10.1007 / \mathrm{s} 003960100508$
(12) Mu, J. H.; Li, G. Z.; Jia, X. L.; Wang, H. X.; Zhang, G. Y. J. Phys. Chem. B 2002, 106, 11685. doi: 10.1021/jp014096a

(13) Mu, J. H.; Li, G. Z.; Wang, Z. W. Rheol. Acta 2002, 41, 493. doi: 10.1007/s00397-002-0246-y

(14) Mu, J. H.; Li, G. Z.; Wang, Z. W.; Zheng, L. Q.; Liao, G. Z.; Huang, L. J. Disper. Sci. Technol. 2001, 22, 421. doi: 10.1081/ DIS-100107851

(15) Acharya, D. P.; Kunieda, H.; Shiba, Y.; Aratani, K. J. Phys. Chem. B 2004, 108, 1790.

(16) Song, B. L.; Hu, Y. F.; Zhao, J. X. J. Colloid Interface Sci. 2009, 333, 820. doi: 10.1016/j.jcis.2009.02.030

(17) Song, B. L.; Hu, Y. F.; Song, Y. M.; Zhao, J. X. J. Colloid Interface Sci. 2010, 341, 94.

(18) Israelachvili, J. N.; Mitchell, D. J.; Ninham, B. W. Journal of the Chemical Society-Faraday Transactions II 1976, 72, 1525. doi: 10.1039/f29767201525

(19) Xie, D. H.; Zhao, J. X. Langmuir 2013, $29,545$.

(20) Granek, R.; Cates, M. E. J. Chem. Phys. 1992, 96, 4758. doi: $10.1063 / 1.462787$

(21) Oda, R.; Narayanan, J.; Hassan, P. A.; Manohar, C.; Salkar, R. A.; Kern, F.; Candau, S. J. Langmuir 1998, 14, 4364. doi: $10.1021 / 1 a 971369 \mathrm{~d}$

(22) Raghavan, S. R.; Kaler, E. W. Langmuir 2001, 17, 300. doi: 10.1021/la0007933

(23) Khatory, A.; Lequeux, F.; Kern, F.; Candau, S. J. Langmuir 1993, 9, 1456. doi: 10.1021/la00030a005

(24) Shrestha, R. G.; Shrestha, L. K.; Aramaki, K. J. Colloid Interface Sci. 2007, 311, 276. doi: 10.1016/j.jcis.2007.02.050

(25) Candau, S. J.; Hirsch, E.; Zana, R.; Delsanti, M. Langmuir 1989, 5, 1225. doi: 10.1021/la00089a018

(26) Fischer, P.; Rehage, H. Langmuir 1997, 13, 7012. doi: 10.1021/ la970571d 